Revue d'histoire de l'Amérique française

RAS REVUE D.HISTOIRE DE L'AMÉRIQUE FRANÇAISE

\title{
Ni janséniste, ni gallican, ni ultramontain : François de Laval
}

\section{Pierre Hurtubise}

Volume 28, numéro 1, juin 1974

URI : https://id.erudit.org/iderudit/303326ar

DOI : https://doi.org/10.7202/303326ar

Aller au sommaire du numéro

\section{Éditeur(s)}

Institut d'histoire de l'Amérique française

\section{ISSN}

0035-2357 (imprimé)

1492-1383 (numérique)

Découvrir la revue

\section{Citer cet article}

Hurtubise, P. (1974). Ni janséniste, ni gallican, ni ultramontain : François de Laval. Revue d'histoire de l'Amérique française, 28(1), 3-26.

https://doi.org/10.7202/303326ar d'utilisation que vous pouvez consulter en ligne.

https://apropos.erudit.org/fr/usagers/politique-dutilisation/ 


\title{
NI JANSÉNISTE, NI GALLICAN, NI ULTRAMONTAIN: FRANÇOIS DE LAVAL *
}

\author{
Pierre Hurtubise \\ Faculté de théologie \\ Université Saint-Paul \\ Ottawa
}

Lorsque le 10 février 1673 Louis XIV publie sa déclaration sur l'extension de la régale, François de Laval, évêque de Pétrée, se trouve à Paris où il s'évertue, depuis quelques mois déjà, à obtenir du pape la création en sa faveur d'un évêché à Québec. La déclaration sur la régale ne suscite pour le moment aucune réaction à Rome ${ }^{1}$, mais la longueur et la complexité des négociations engagées entre Paris et la cour pontificale au sujet du siège épiscopal de Québec - elles sont en cours depuis au moins $1662^{2}$ - suffisent, à elles seules, à démontrer à quel point sont laborieuses, à l'époque, les relations entre les deux puissances.

Ne nous attardons pas, pour le moment, sur le détail de ces affrontements. Nous savons qu'ils ont existé et qu'ils ont eu une profonde influence sur la vie religieuse, à vrai dire, sur la vie tout court en France dans la deuxième moitié du XVIIe siècle. En a-t-il été de même pour la Nouvelle-France ? Si oui, comment ces affrontements y ont-ils été connus et percus ? Quelles réactions y ont-ils suscitées? Quelles prises de position, déterminées? C'est ce que nous allons demander à un témoin privilégié de ces événements, celui en tout cas qui était en NouvelleFrance le plus immédiatement concerné par eux et le plus exposé à en subir les contre-coups, François de Montmorency-Laval, vicaire apostolique de Nouvelle-France et premier évêque de Québec.

* Version revue et augmentée d'un texte présenté au colloque sur "le monde français en 1673", tenu à l'Université Queen's (Kingston) en octobre 1973.

${ }^{1} \mathrm{P}$. Blet, Les Assemblées du clergé et Louis XIV (1670-1693) (Rome 1972), 74-76.

2 M. E. Faillon, Histoire de la colonie française en Canada, III (Villemarie [i.e. Montréal] 1866) : 427. - A. Vachon, "Laval, François de", dans Dictionnaire biographique du Canada, II (Québec-Toronto 1969) : 382-383.

RHAF, vol. 28, no 1 (juin 1974) 


\section{L'homme}

Tout d'abord, quelques mots du personnage. Né à Montignysur-Avre, dans le Perche, en 1623, François de Laval, comme la plupart des prélats français de son époque, appartient à la noblesse: noblesse d'épée dans le cas de son père, Hugues de Laval, issu d'une branche cadette de la célèbre famille des Montmorency ${ }^{3}$; noblesse de robe dans le cas de sa mère, Michelle Péricard, fille et petite-fille de parlementaires rouennais ${ }^{4}$.

Il restera toute sa vie marqué par le poids de ses antécédents. La piété, le zèle et l'esprit de renoncement dont il fera preuve plus tard, au témoignage unanime de ses contemporains, n'arriveront jamais à faire disparaître chez lui un certain entêtement que Marie de l'Incarnation appelle "inflexibilité" et François de la Colombière, son panégyriste, "courage", entêtement qu'ils mettent tous deux au compte de son zèle épiscopal ${ }^{5}$, mais qui pourrait bien être plutôt la rançon de son tempérament et de ses convictions aristocratiques. L'intransigeance avec laquelle il fait supprimer les manifestations populaires de la Saint-Jean en 1654, dans sa seigneurie de Montigny ${ }^{6}$, ne fait qu'un avec l'attitude cassante qu'il adoptera plus tard à Québec dans l'exercice de ses fonctions épiscopales ${ }^{7}$. C'est peut-être le seul trait qui le rattache vraiment à son milieu d'origine - tout le reste, nous le verrons plus loin, semble avoir été puisé ailleurs -, mais c'est un trait psychologique important, qui colore toute sa personnalité, et, à ce titre, il mérite d'être retenu.

En 1631, Francois de Laval quitte Montigny pour La Flèche où sa famille l'envoie faire ses humanités ${ }^{8}$. Il n'a alors que huit ans. Ce départ marque le début d'un long périple intellectuel et spirituel qui va le conduire de La Flèche à Paris, puis de Paris à Caen, le mettre en contact avec des milieux totalement gagnés à l'idéal d'une réforme mystique et morale du royaume et faire

${ }^{3} \mathrm{~A}$. Gosselin, Vie de Mgr de Laval, premier évêque de Québec et apôtre du Canada, 1622-1708, I (Québec 1890): 7-9.

4 Ibid.: 10. H. de Frondeville, Les conseillers du Parlement de Normandie au XVIe siècle (Rouen-Paris 1960), 502.

5 Quebecen., Beatificationis et Canonizationis Ven. Servi Dei Francisci de Montmorency-Laval, Primi Episcopi Quebecensis, Altera Nova Positio Super Virtutibus, II (Rome 1956) : 54, 59, 638. Nous utiliserons désormais le titre court Altera Nova Positio pour désigner cet important recueil de documents.

6 Ibid.: 15-16.

7 Ibid.: $41-59 ; 251-335$ et passim.

8 A. Vachon, art. cit.: 374 . Sur le collège de La Flèche, cf. C. de Rochemonteix, Un collège de Jésuites aux XVIIe et XVIIIe siècles: le collège Henri IV de La Flèche, 2 vol. (Le Mans 1889). 
de lui ce qu'il est convenu d'appeler à l'époque un dévot. Deux présences dominent de loin cet itinéraire: celle du jésuite Jean Bagot, directeur de la Congrégation mariale du collège de La Flèche, puis fondateur de l'Aa (ou Société des Bons Amis) de Paris, dont François de Laval fera son père spirituel et sous lequel il s'initiera, dans l'une et l'autre de ces pieuses associations, à la vie intérieure et à la vie apostolique ${ }^{9}$; celle du laïque Jean de Bernières, fondateur de l'Ermitage de Caen, mystique engagé, dont le futur évêque de Québec fera son modèle et auprès duquel il achèvera de se dépouiller de toute attache aux biens et aux maximes de ce monde ${ }^{10}$.

François de Laval gardera toute sa vie un souvenir ému de ces deux hommes et un attachement indéfectible aux milieux et aux idéaux qu'ils représentaient. Ses anciens maîtres jésuites le savaient bien, eux qui, dès 1653 , chercheront à le faire nommer vicaire apostolique du Tonkin ${ }^{11}$, puis, cinq ans plus tard, s'emploieront, avec succès cette fois, à lui obtenir le vicariat apostolique de Nouvelle-France ${ }^{12}$. Ses anciens condisciples de l'Aa: un Pierre Lambert de la Motte, un Vincent de Meur, un François Pallu, pour ne citer que ceux-là, le savaient également et lorsque un certain nombre d'entre eux jetteront les bases du Séminaire des Missions-Etrangères vers 1658, ils prendront soin de l'associer à leur entreprise et d'établir avec lui des liens matériels et spirituels durables ${ }^{13}$. Ses anciens confrères de la Compagnie du Saint-Sacrement - car il avait été des leurs à titre de membre de l'Ermitage ${ }^{14}$ - le savaient aussi, comme en témoigne l'intérêt spécial qu'ils porteront, dès le départ, à ses travaux apostoliques en Nouvelle-France ${ }^{15}$.

Ces diverses allégeances peuvent déjà servir à définir les paramètres spirituels à l'intérieur desquels le futur évêque de Québec tend à se mouvoir. François de Laval est, d'abord et

${ }^{9}$ A. Vachon, art. cit.: 375. A. Gosselin, op. cit., I: 30-31. R. Daeschler, "Bagot, Jean", dans Dictionnaire d'histoire et de géographie ecclésiastiques, VI (1932), col. 223-224. A. A. de Backer, C. Sommervogel, Bibliothèque de la Compagnie de Jésus, I (Paris-Bruxelles 1890) : col. 774-777.

$10 \mathrm{M}$. Souriau, Le mysticisme en Normandie au XVIIe siècle (Paris $1923)$, 365 et suiv.

11 H. Chappoulie, Aux origines d'une Eglise. Rome et les missions d'Indochine au XVIIe siècle, I (Paris 1943) : 102 et suiv.

12 Altera Nova Positio: 16-27. A. Vachon, art. cit.: $375-377$.

13 G. de Vaumas, L'éveil missionnaire de la France (Lyon 1942), 399 et suiv., N. Baillargeon, Le Séminaire de Québec sous l'épiscopat de Mgr de Laval (Québec 1972), 11 et suiv.

14 M. Souriau, op. cit., 42 et suiv.

15 R. Allier, La Compagnie du Très Saint-Sacrement de l'Autel. La "Cabale des Dévots" 1627-1666 (Paris 1902), 149-150. 
avant tout, un dévot hanté par l'idée de la conversion des infidèles - ce qui explique son départ pour la Nouvelle-France -, mais en même temps profondément marqué par le rigorisme de l'époque et par le mythe d'un retour à une sorte de société chrétienne idéale - d'où l'intransigeance de ses interventions, comme évêque, dans des domaines que d'aucuns, même à l'époque, estimaient ne pas être de sa compétence.

En cela, il faut le dire, il ne se distingue pas tellement d'une bonne partie de l'épiscopat de son temps. En cette deuxième moitié du XVIIe siècle, en effet, le mouvement de réforme tridentine a commencé à produire en France ses effets et, malgré les aléas du système existant de nominations aux bénéfices, il ne manque pas dans le royaume de prélats zélés qui n'ont peutêtre pas le tonus spirituel de François de Laval, mais qui n'en partagent pas moins avec lui un certain nombre de convictions concernant le "rétablissement" de la religion en France.

\section{Le milieu épiscopal}

Pierre Blet a tracé de ce corps épiscopal un portrait très exact et très nuancé qu'il est peut-être bon de rappeler ici pour bien replacer François de Laval dans son cadre social et idéologique propre.

Première constatation: ces évêques ont une conscience très aiguë de leurs droits et de leurs privilèges et ils sont convaincus de la prééminence du rôle qu'ils sont appelés à jouer dans le royaume. Ils sont, bien entendu, sujets du roi, mais ils veulent ne dépendre "que du Roi seul", tous "les officiers du prince" devant, selon eux, "céder le pas devant les ministres du Seigneur". Et ces principes, ils voudraient les voir appliquer partout, en toutes circonstances, "jusque dans les détails d'une cérémonie funèbre ${ }^{16 "}$. D'où les nombreuses querelles de préséance, les nombreux accrochages avec les autorités civiles qu'ils suscitent eux-mêmes ou auxquels ils se trouvent par la force des choses mêlés. François de Laval pourrait servir ici presque de modèle du genre ${ }^{17}$.

Ajoutons que chez ces prélats-gentilshommes la fierté du passé et le prestige du présent ne sont pas sans affecter la conception qu'ils se font d'eux-mêmes et de leur rang. Ils ne peuvent oublier que leurs "aïeux ont gouverné des comtés et des duchés" et que leurs frères et cousins "commandent aujour-

16 P. Blet, Les Assemblées du clergé, 582.

17 Altera Nova Positio: 43-51, 180-181, 216-218, 349-369. 
d'hui les régiments du Roi ${ }^{18}$ ". Mais on ne peut nier ce fait qu'ils ont en même temps le souci réel et sincère de remplir leur fonction de successeurs des Apôtres.

Leur grande préoccupation au XVIIe siècle a été la foi catholique - une foi à restaurer dans l'unité et la vérité, contre les ennemis du dehors aussi bien que contre ceux du dedans. Dans ce combat pour l'orthodoxie, ils se sentent solidaires de l'évêque de Rome ${ }^{19}$. Car, comme le souligne si justement le P. Blet,

c'est bien l'unité catholique et romaine que le Roi et les évêques entendent restaurer. Un Bossuet, un Harlay, un Le Tellier reconnaissent dans l'évêque de Rome le centre de l'unité, le suprême gardien de la foi et de la discipline, le successeur du prince des apôtres, et le vicaire du Christ par excellence. Ils ne dénient pas à chaque évêque le droit de porter jusqu'à Rome ses causes et ses doléances, car ils comprennent que son autorité est garante de la leur. Ils reconnaissent dans le Siège Apostolique la citadelle de la liberté ecclésiastique

et ils avouent franchement qu'il n'est pas moins de leur intérêt que de leur devoir de défendre et de maintenir cette dernière ${ }^{20}$.

Sans doute, ont-ils sur leurs propres pouvoirs, sur ceux du pape et sur leurs rapports avec ce dernier, des vues qui ne concordent pas toujours avec celles de Rome, d'où les conflits qui les opposent, tout au long du XVIIe siècle, au Siège Apostolique - nous y reviendrons plus loin -, mais ces manifestations d'indépendance ne les empêchent pas de proclamer tout haut, chaque fois qu'ils en ont l'occasion, leur respect pour "l'auguste et [...] sacrée autorité que Jésus-Christ a donnée à ses vicaires en [sur] terre ${ }^{21}$ ". Tout comme leurs remontrances au roi - et ils sont, en ce domaine, parfois aussi audacieux à l'endroit de ce dernier qu'à l'endroit du pape - n'impliquent en rien leur rejet de la monarchie ou la remise en cause des prérogatives royales ${ }^{22}$.

Car, malgré toutes leurs audaces et en dépit de toutes leurs susceptibilités, les évêques gallicans ont pour l'une et l'autre de ces puissances le même respect, la même vénération. L'évêque de Rome est leur chef au spirituel, le roi l'est au temporel; le premier, vicaire du Christ dans les choses spirituelles, le second, vicaire de Dieu dans les choses temporelles; l'un et l'autre por-

$18 \mathrm{P}$. Blet, Les Assemblées du clergé, 582.

19 Ibid., 582-583.

20 Ibid., 583.

21 Ibid., 380.

22 P. Blet, Le clergé de France et la monarchie, II (Rome 1959) : 400. 
tant "sur leur visage le reflet de la majesté divine"; l'un et l'autre dépositaires d'une puissance, non pas oppressive, mais tutélaire et complémentaire de leur propre autorité ${ }^{23}$.

Mais, inévitablement, des conflits surgissent, des oppositions se font jour, qui viennent briser, momentanément du moins, l'harmonie que l'on souhaiterait voir régner entre l'une et l'autre de ces puissances, et les prélats ne peuvent éviter, bien plus se trouvent chaque fois devant l'obligation de prendre parti. Ils le font habituellement tous en faveur du roi. Par intérêt ? Par crainte servile, comme les en accusera le bref Paternæ Charitati en $1682^{24}$ ? Les choses ne sont pas aussi simples que cela.

On peut taxer de flatterie nombre d'éloges adressés au roi, en particulier à l'occasion des Assemblées du clergé; on peut même croire que les évêques n'avaient pas la force morale de résister à un maître qui était, en même temps que leur roi, le patron de tous les riches bénéfices du royaume. Il semble toutefois que le cardinal de Bouillon soit plus près de la vérité lorsqu'il explique en 1682 au cardinal Cibo que

les membres de l'Assemblée n'ont pas eu le courage de s'opposer à une chose que la majeure partie désapprouvait en son cœur - c'est-à-dire la Déclaration sur la régale - en partie par la soumission (qu'ils avaient) envers la volonté du roi, et en partie par le désir de plaire à un prince dont les grandes qualités mériteraient l'adoration du monde entier, s'il était permis d'adorer quelque chose sur terre ${ }^{25}$.

C'est donc d'abord et avant tout par respect qu'ils agissent ainsi, par respect et par vénération pour un homme qui est, à leur yeux, l'image vivante de la divinité et auquel ils reconnaissent, sur ses sujets, une puissance presque sacerdotale ${ }^{26}$; un homme, par surcroît, auquel l'Eglise de France doit beaucoup.

Ce faisant, ni eux ni le roi ne songent un seul instant à une rupture définitive avec Rome. La preuve, c'est que tout en appuyant leur roi, les évêques s'emploient généralement en coulisse à ménager des terrains d'entente entre lui et le pape, tout comme, à l'inverse, le roi n'hésite pas, le cas échéant, à se faire "conciliateur et arbitre entre le Pape et les évêques ${ }^{27}$ ".

${ }^{23}$ Id., Les Assemblées du clergé, 586-587.

24 Ibid., 363-370.

25 Ibid., 588.

$26 \mathrm{P}$. Blet, Le clergé de France, II : 422-423.

27 Id., Les Assemblées du clergé, 65. 
Cet épiscopat gallican qu'on représente si souvent comme courtisan ou, au contraire, comme rebelle, ne correspond en réalité ni à l'un ni à l'autre de ces qualificatifs. Il tient à son rang et à ses prérogatives et il les défend jalousement chaque fois que les circonstances l'exigent. Mais il sait en même temps à quel point sa propre autorité est liée à celle du pape et du roi et à quel point il ne peut rompre avec l'une ou l'autre de ces puissances sans attenter à ses propres pouvoirs et à sa propre existence ${ }^{28}$. C'est ce que Harlay de Champvallon, archevêque de Paris, rappellera à ses collègues en 1681 en leur disant que "le Clergé de France ayant toujours conservé un grand respect pour le Saint Siège, une fidélité inviolable au Roi, une fermeté inébranlable pour la conservation des droits et des libertés de l'Eglise gallicane, il fallait demeurer dans cet esprit, qui avait rendu l'Eglise de France auguste ${ }^{29}$ '.

Telle est, en gros, l'image qui semble se dégager des plus récentes études entreprises sur l'épiscopat français au XVIIe siècle. Jusqu'à quel point François de Laval correspond-il à ce stéréotype ? Jusqu'à quel point s'en éloigne-t-il ? C'est la question à laquelle nous voudrions maintenant essayer d'apporter une réponse.

\section{Indices et circonstances}

Signalons tout d'abord les difficultés de l'entreprise. Une première tient à la personnalité même de François de Laval. N'ayant le goût ni de la théorie ni de la spéculation - ses préoccupations sont nettement d'un autre ordre - il ne nous a laissé que peu de textes, peu de témoignages relatifs aux grandes questions qui étaient débattues en son temps. Rien sur le jansénisme, très peu sur le gallicanisme, guère plus sur l'ultramontanisme.

Une deuxième difficulté, c'est que, par un étrange concours de circonstances, il n'a jamais été appelé à se soumettre aux différents tests d'orthodoxie imposés au clergé gallican dans la deuxième moitié du XVIIe siècle. Le formulaire anti-janséniste de 1657, qui le trouve à l'Ermitage de Caen, c'est-à-dire dans le diocèse de Bayeux, ne sera promulgué dans ce diocèse qu'une année plus tard, soit au moment où François de Laval avait quitté l'Ermitage à la suite de sa nomination comme vicaire

28 Ibid., 588.

29 Ibid., 233. 
apostolique de Nouvelle-France ${ }^{30}$. Cette dernière commission, qui le rattachait directement à Rome, lui vaudra d'échapper également au formulaire de 1661, destiné à tous les ecclésiastiques, régents, maîtres d'école, religieuses et bénéficiers du royaume, de même qu'à la bulle et au formulaire de $1665{ }^{31}$. Il ne lui sera pas demandé non plus de se soumettre à l'Edit de la régale $(1673,1675)$ ni aux célèbres Quatre articles gallicans (1682), le roi ayant omis, dans l'un et l'autre cas, de les faire enregistrer par le Conseil Souverain ${ }^{32}$.

Le cas du formulaire de 1657 excepté, toutes ces anomalies s'expliquent par le fait très simple que la Nouvelle-France se trouve dans une situation assez particulière par rapport à la métropole. Soumise directement au roi, même sous le régime des Compagnies, la colonie canadienne est dotée d'un appareil administratif qui, dans sa structure, sinon dans son fonctionnement, ressemble beaucoup à celui des provinces de France, mais avec des différences importantes résultant, en grande partie, des circonstances particulières - dimensions du territoire, éloignement de la métropole, etc. - dans lesquelles se trouve placée la Nouvelle-France ${ }^{33}$. Ce qui explique que nombre d'édits, d'ordonnances et de déclarations de la cour ne sont pas enregistrés dans la colonie, dans certains cas, parce qu'ils ne s'y appliquent pas; dans d'autres, probablement parce que le roi et ses ministres n'estiment cette formalité ni utile ni indispensable au bon gouvernement de la colonie canadienne ${ }^{34}$.

La Nouvelle-France n'est pas la France. La preuve, c'est qu'en 1687, la cour n'hésitera pas un seul instant à solliciter les bulles de Mgr de Saint-Vallier, nouvel évêque de Québec, alors que, cinq ans plus tôt, le roi, sur les conseils du cardinal d'Estrée, 109.

${ }^{30} \mathrm{H}$. Fisquet, La France pontificale. Bayeux et Lisieux (Paris (s.d.)),

31 Sur ces bulles et formulaires, voir les Mémoires du clergé, I (Paris 1768) : col. 349 et suiv.

32 Altera Nova Positio: 951.

33 Voir A. Vachon, "L'administration de la Nouvelle-France", dans Dictionnaire biographique du Canada, II (Québec-Toronto 1969): XV et suiv., W. J. Eccles, "Buade de Frontenac et de Palluau, Louis de", Ibid. (Québec-Toronto, 1966), I: 138-139.

34 Alors que l'Edit sur l'extension de la régale (1673) et d'autres de la même importance ne sont pas enregistrés en Nouvelle-France, des règlements d'une portée beaucoup plus limitée le sont, tels l'Edit contre les blasphémateurs $(1666)$ et celui contre les duels (1679). Voir, à ce propos, l'Inventaire des insinuations du Conseil Souverain de la Nouvelle-France (P.-G. Roy, Beauceville 1929), 21, 69. Simple effet du hasard ou indice d'une politique d'adaptation aux réalités de la colonie ? La deuxième explication paraît plus vraisemblable. 
avait pris la ferme résolution de ne présenter aucun candidat à Rome, tant que le pape n'aurait pas rappelé son veto contre les prélats qui avaient participé à l'Assemblée de 1682, et que, le cas de Québec excepté, cette décision sera scrupuleusement respectée jusqu'en 1692, c'est-à-dire jusqu'à ce qu'un arrangement intervienne entre Rome et Paris à ce sujet ${ }^{35}$.

La situation juridique dans laquelle se trouve François de Laval, soit comme vicaire apostolique de 1658 à 1674, soit comme évêque de Québec à partir de cette dernière date, diffère, elle aussi, passablement de celle de l'ensemble des évêques du royaume. Il est, comme eux, nommé par la cour - sur ce point Rome a accepté de respecter le droit concordataire ${ }^{36}$-, il prête comme eux serment de fidélité au roi ${ }^{37}$, mais ni en 1658 ni en 1674 peut-il être considéré comme faisant partie de l'organisation de l'Eglise gallicane. A titre de vicaire apostolique, nous l'avons vu, il relève directement du Saint-Siège; à titre d'évêque de Québec, il va se trouver dans une sorte de no man's land ecclésiastique, Rome ayant obtenu que Québec ne soit rattaché à aucune des métropoles françaises existantes ${ }^{38}$.

Une des conséquences de tout ceci, c'est que François de Laval, bien que faisant partie de l'épiscopat gallican, bien que lié, par amitié autant que par intérêt, avec un certain nombre de prélats du royaume ${ }^{39}$, ne participe pas aux activités comme telles de l'Eglise gallicane, n'a le droit ni de siéger ni de se faire représenter aux Assemblées du clergé et se trouve, par le fait même, plus ou moins coupé des grands débats et des grandes controverses de l'heure. Comment se surprendre après cela du peu d'intérêt que ces événements suscitent en Nouvelle-France et du silence presque total dont François de Laval semble les avoir entourés avant comme après 1674 ?

Les témoignages directs faisant défaut, pour les raisons que nous venons d'exposer, force nous est de recourir à des indices d'ordre plus circonstanciel. Lesquels ? Ceux précisément que permet d'atteindre le biais des personnes et des milieux fréquentés par François de Laval, aussi bien pendant ses années de formation qu'au cours de ses années d'épiscopat. Les biographes du premier évêque de Québec qui se sont livrés à cette étude ont cru

35 P. Blet, Les Assemblées du clergé, 414-415; 540 et n. 30.

36 Altera Nova Positio: XXV.

37 Ibid.: 348.

38 Ibid.: 335-338.

39 Entre autres, l'archevêque de Paris. Voir, à ce sujet, Altera Nova Positio: 175, 177-179, 438. 
pouvoir en conclure, se fondant sur l'adage bien connu: Qui se ressemble s'assemble, que François de Laval était, à l'image des maîtres et des milieux dont il se réclamait, un ennemi juré du jansénisme et un allié inconditionnel de Rome. Ils ont été en général suivis sur ce point et on pourrait peut-être encore aujourd'hui être tenté d'accepter d'emblée une telle conclusion, n'était la fragilité, de plus en plus apparente, des bases sur lesquelles elle repose.

Le P. Blet a fait justice, il y a quelques années, de l'affirmation quelque peu simpliste selon laquelle l'escadron jésuite français, au XVIIe siècle, emboîtait unanimement le pas, donné par Rome, de l'ultramontanisme. Soupçonnés d'enseigner et de soutenir la thèse du régicide - ce qui leur avait valu les ennuis que l'on sait à la fin du XVIe siècle -, les Jésuites français étaient devenus, au XVIIe, extrêmement prudents en ces matières, évitant soigneusement toute manifestation qui eût pu les rendre de nouveau suspects au roi et aux Parlements. Ainsi, au temps où François de Laval faisait sa théologie à Clermont, était-il interdit aux professeurs de ce collège de discuter, voire même d'aborder devant leurs élèves ces questions "délicates" ${ }^{40}$. Les Jésuites étaient, par ailleurs, nombreux à admettre avec le $P$. de La Chaize *, confesseur de Louis XIV, la doctrine de l'indépendance absolue du roi au temporel, telle que consignée dans le premier des Quatre articles gallicans. Ils partageaient en cela le sentiment unanime de l'ensemble du clergé et de la société française, dont, soulignons-le, on a eu trop souvent tendance à les isoler. Bons Français, ils entendaient, eux aussi, en délimitant les domaines du temporel et du spirituel, faire suffisamment "belle la part du Roi et du pouvoir civil ${ }^{41 "}$.

Antijansénistes, les Jésuites l'étaient, si l'on veut entendre par là qu'ils étaient en guerre ouverte avec le parti et un certain nombre d'idées jansénistes. Le P. Jean Bagot, dont il a été question plus haut, était, vers 1650, un des Jésuites les plus actifs en ce domaine. Mais si l'on en juge par le principal combat qu'il livra aux jansénistes, c'est-à-dire la campagne qu'il entreprit contre eux au sujet des privilèges des réguliers, privilèges contre lesquels Saint-Cyran s'était élevé au nom des droits prioritaires de l'épiscopat ${ }^{42}$, force nous est d'admettre qu'il y avait plusieurs

$40 \mathrm{G}$. Dupont-Ferrier, Du collège de Clermont au lycée Louis-le-Grand (1563-1920), I: 170-171.

* Ou de La Chaise.

41 P. Blet, "Jésuites gallicans au XVIIe siècle", dans Archivum Historiae Societatis Jesu, XXIX (1960): 82.

$42 \mathrm{Cf}$. A. A. de Backer, C. Sommervogel, Bibliothèque de la Compagnie de Jésus, I : col. 774-776. 
types d'antijansénisme à l'intérieur de la Compagnie, dont certains avaient assez peu à voir avec le jansénisme proprement dit. Sur le point précis qui opposait Jean Bagot à Saint-Cyran, en tout cas, on est en droit de penser que François de Laval, neveu d'évêque et futur évêque lui-même, devait se sentir plus près du "janséniste" que du "jésuite".

Jean de Bernières a été lui aussi présenté comme un ennemi juré des jansénistes. C'est un fait qu'il a été l'objet de violentes attaques de leur part et qu'il a eu à leur endroit des paroles extrêmement dures. Mais en quoi au juste leur était-il hostile ? Encore ici, il est curieux de noter que les conflits portent rarement sur des points de doctrine, mais qu'il semble toujours s'agir de luttes d'influence, d'intrigues dont l'enjeu est, la plupart du temps, le contrôle spirituel d'un groupe, d'une communauté, d'un couvent ${ }^{43}$. On en vient à se demander s'il ne s'agirait pas, dans la plupart de ces cas, de problèmes d'appartenance beaucoup plus que de croyance.

\section{Idéologies et personnes}

Tout ceci nous ramène à un autre problème, beaucoup plus fondamental, que nous avons laissé de côté jusqu'ici, mais que nous ne pouvons esquiver plus longtemps, si nous voulons arriver à situer vraiment François de Laval par rapport aux hommes et aux idées de son temps. C'est celui de savoir ce que des mots comme gallicanisme, jansénisme, ultramontanisme peuvent bien signifier lorsque appliqués aux réalités du XVIIe siècle.

Ces néologismes sont, nous le savons, des catégories commodes créées, dans le cas du couple gallicanisme-ultramontanisme, au XIXe siècle ${ }^{44}$, dans le cas du mot jansénisme, vers $1650^{45}$, mais ayant subi, depuis, de nombreux avatars aux mains des théologiens, historiens ou controversistes qui les ont utilisés. Jusqu'à quel point ces vocables et leurs définitions successives correspondent-ils à la réalité du XVIIe siècle ? Jusqu'à quel point la trahissent-ils ? C'est la question que nous ne pouvons éviter de nous poser.

Comme le rappelle très justement le chanoine Martimort, le XIXe siècle entendait par gallicanisme "la doctrine de la Déclaration du clergé de 1682, jugée à la lumière de tous les événe-

43 M. Souriau, Le mysticisme en Normandie, 297 et suiv.

44 Y. Congar, "Gallicanisme", dans Catholicisme, IV : col. 1731.

43 A.-G. Martimort, Le gallicanisme de Bossuet (Paris 1953), 13. 
ments survenus depuis, au XVIIIe siècle et dans l'atmosphère des polémiques qui préparaient le Concile Vatican 46". C'est cette définition que les biographes de François de Laval ont généralement à l'esprit lorsqu'ils s'emploient à disculper leur héros de l'accusation de gallicanisme. Au XVIIe siècle, le mot n'existe pas, parce que les idées et les pratiques qu'on appellera plus tard gallicanes ne constituent pas, à l'époque, "un système susceptible d'être enfermé dans des formules précises, un corps cohérent et homogène de doctrines [...]. Il y a divers courants d'opposition au Saint-Siège, différents les uns des autres par leur origine, leur ancienneté, leur importance, indépendants dans leur évolution ${ }^{47}$ ".

Dans une tentative de ramener cette diversité à un commun dénominateur, Victor Martin a cru pouvoir décrire le gallicanisme comme un "accord du roi et du clergé pour gouverner l'Eglise de France en contrôlant et refrénant l'ingérence du Saint-Siège", mais avec "la volonté délibérée de ne pas rompre avec l'Eglise romaine ${ }^{48}$ ". Définition qui n'est pas sans mérites, mais qui, de fait, laisse trop d'éléments de côté pour pouvoir rendre compte de la variété des formes de gallicanisme qui ont existé en France au XVIIe siècle.

Aussi certains proposent-ils de distinguer, pour cette époque, deux grands types de gallicanisme, l'un, politique, qui viserait à assurer "l'indépendance totale du pouvoir royal à l'égard de l'autorité spirituelle du pape et la plénitude de l'autorité royale sur la vie concrète et l'administration de l'Eglise"; l'autre, ecclésiastique, qui, tout en acceptant les conséquences d'un certain "absolutisme" royal, s'emploierait plutôt à la défense des droits du clergé face aux empiétements des pouvoirs aussi bien civil que pontifical ${ }^{49}$. D'autres voudraient que l'on distingue à l'intérieur du gallicanisme politique au moins deux formes de gallicanisme: l'une, plus rigide, parce que plus théorique, que l'on appellerait le gallicanisme parlementaire; l'autre, plus souple et plus pratique, à laquelle on donnerait le nom de gallicanisme royal ${ }^{50}$.

Il nous semble qu'il vaudrait mieux retenir, pour l'époque - quitte à apporter les nuances voulues dans chaque cas individuel -, deux formes-types de gallicanisme, l'une que l'on

46 Ibid., 13-14.

47 Ibid., 14.

48 V. Martin, Les origines du gallicanisme, I (Paris 1939) : 31.

49 Y. Congar, art. cit.: col. 1734.

50 M. Dubruel, "Gallicanisme", dans Dictionnaire de théologie catholique, XXII : col. 1125-1126. 
pourrait appeler idéologique ou doctrinale; l'autre que l'on appellerait tout simplement pratique ou pragmatique: la première, représentée par les théologiens et les parlementaires, théoriciens les uns et les autres, chacun à leur façon, des "libertés gallicanes"; la seconde, incarnée dans le roi, ses ministres et l'épiscopat, practiciens, à divers degrés, de ces mêmes "libertés", mais pour autant que leurs intérêts, d'ordre surtout juridictionnel, les y poussent. Ce qui ne nous empêchera pas de reconnaître que ces "practiciens" utilisent assez souvent les arguments des "théoriciens", mais que, s'ils le font, c'est très souvent pour des raisons de tactique, voire même d'expédient, plutôt que par souci d'exploiter la valeur en soi de ces arguments.

L'affaire de la régale nous fournit de cette attitude une parfaite illustration. Louis XIV entendait régler cette question conformément aux vœux exprimés par ses conseillers et par les Parlements, mais alors que ces derniers en faisaient une affaire de principe, lui ne s'intéressait, à vrai dire, qu'aux avantages politiques qu'il comptait pouvoir en tirer. Cela est si vrai que "devant les remontrances du Pape, il se déclare prêt à accepter un indult pour exercer le droit de régale, ce qui est la négation même de la théorie parlementaire de la régale, droit inné et imprescriptible de la Couronne ${ }^{51}$ ". D'autre part, aux évêques qui se déclarent opposés au principe même de la régale, il fait toute une série de concessions qui réduisent à tel point les inconvénients qu'ils redoutaient de cette innovation, qu'ils finissent par se rallier à son point de vue ${ }^{52}$. Preuve qu'ils savent, eux aussi, oublier les principes lorsque leurs intérêts et ceux de l'Eglise gallicane sont en jeu.

Malheureusement pour eux, le pape Innocent XI n'est pas aussi conciliant, et c'est son refus obstiné qui va provoquer l'Assemblée extraordinaire de 1681-82, puis, éventuellement, la formulation des Quatre articles. La publication de ces derniers pourrait donner à penser que le roi et les évêques s'étaient finalement ralliés au point de vue des idéologues gallicans. Il n'en est rien. Nous savons aujourd'hui que c'est la rumeur d'une censure pontificale imminente qui convainquit Louis XIV de hâter la discussion et la publication des Quatre articles. Et il semble bien que son intention était de n'utiliser ce texte que comme une arme tactique ou, si l'on préfère, un épouvantail. "La fausse alarme dissipée, constate le P. Blet, [Louis XIV] n'apparaît pas un défenseur très convaincu des Quatre arti-

${ }^{51} \mathrm{P}$. Blet, Les Assemblées du clergé, 594.

52 Ibid., 259 et suiv. 
cles ${ }^{53}$." Tout d'abord, il s'empresse de renvoyer les évêques dans leurs diocèses. Plus tard, bien qu'il s'oppose à une rétractation pure et simple de la doctrine des Quatre articles - ce que Rome d'ailleurs ne demande pas, comme on s'en rendra compte en $1692^{54}$ - il se déclare tout prêt à la laisser sombrer dans l'oubli.

Je ne puis rien changer, écrit-il en 1689 à son ambassadeur à Rome, à tout ce qui s'est fait par l'Assemblée du clergé de mon royaume ni donner la moindre atteinte à ma Déclaration survenue en conséquence, et l'on ne doit pas espérer au lieu où vous êtes (Rome) que quelqu'absolue que mon autorité soit dans mon royaume [...] je puisse faire ce que la Cour où vous êtes désirerait

- entendons obtenir une rétractation -, mais il s'empresse d'ajouter que la déclaration nécessaire au temps d'Innocent XI, ne l'est plus en 1689 et que, par conséquent, "tout cet ouvrage ne doit être considéré que comme un boulevard de terre qui s'éboulera facilement et s'aplanira bientôt s'il est négligé de part et d'autre et que la cour de Rome ne songe pas plus à l'attaquer que je ne me mettrai en peine de le défendre quand la bonne intelligence sera rétablie ${ }^{55}$ ".

Cela ne suffisant pas, Louis XIV, nous le savons, finira par consentir, en 1693, à ce que Rome demandait depuis le début: l'annulation pure et simple de la Déclaration de 1682. Fait significatif, les évêques accepteront sans difficulté de signer une rétractation en ce sens préparée par les cours de Rome et de Paris ${ }^{56}$.

La complexité même des mobiles qui sous-tendent toute cette affaire permet de

soupçonner tout ce qu'a d'imparfait un terme comme celui de gallicanisme, appliqué à l'étatisme pratiqué par les magis-

53 Ibid., 596.

54 A Croissy qui reproche, en 1692 , à Rome de demander une rétractation des Quatre Articles, le nonce répond qu' "il y a là une équivoque manifeste, parce que Sa S.teté ne demande pas la rétractation de la doctrine, Elle laisse chacun croire ce que lui dicte sa propre conscience. Mais que plusieurs évêques aient eu l'audace de s'assembler pour publier une déclaration contre l'autorité du Pape, c'est une chose qu'on ne peut excuser d'être un affront manifeste et un manque de respect. On sait bien que ces opinions ont été d'autres fois soutenues et attaquées comme problématiques; mais qu'un petit groupe d'évêques et d'abbés, qui sont évidemment inférieurs, veuillent le déclarer pour doctrine saine et constante et qu'après cela le Pape doive les promouvoir à l'épiscopat sans qu'ils aient rétracté cet acte injurieux, cela paraît vraiment exhorbitant" (Cité par P. Blet, Les Assemblées du clergé, 559).

55 Ibid., 498.

56 Ibid., 568. 
trats en même temps qu'au désir d'autonomie manifesté par les prélats de France. Même en contre-distinguant un gallicanisme parlementaire, un gallicanisme épiscopal et encore un gallicanisme royal, on court le risque de réunir sous un terme unique des réalités opposées ${ }^{57}$.

$\mathrm{Au}$ fond, le roi et les évêques accordent, en général, assez peu d'importance aux spéculations des juristes et des théologiens: ce qui les préoccupe, d'abord et avant tout, c'est la sauvegarde de leurs droits face aux prétentions ultramontaines. Comme se plaît à le répéter Colbert: "Quand le pape est notre ami, il sera infaillible, quand il ne le sera pas, il sera hérétique ${ }^{58}$." Simple boutade, mais qui traduit fort bien l'attitude de la cour.

Les évêques savent qu'en ce domaine, leur cause est solidaire de celle du roi. D'où l'appui qu'ils lui apportent. Mais ils ont aussi des raisons bien à eux d'agir en ce sens, car ils ne peuvent rester insensibles à la menace que semble faire peser sur leur propre pouvoir une interprétation, à leurs yeux, excessive des pouvoirs de l'évêque de Rome. Jaloux de leurs "libertés", ils n'entendent pas être réduits au rang de "simples vicaires du pape, révocables ad nutum ${ }^{59}$ "; au contraire, ils tiennent à conserver à l'épiscopat toute sa grandeur et toute sa dignité.

Chaque évêque, proclame l'Assemblée de 1655 , est le seul vicaire de Jésus-Christ en son diocèse, le seul chef de son Eglise, le premier pasteur de cette portion du troupeau de Notre Seigneur et enfin l'époux du siège qu'il remplit, et [...] il ne peut être dépouillé de l'autorité qu'il a de droit divin sur les ouailles qui lui ont été commises ${ }^{60}$.

Et admettre cela, commente Bossuet en 1681, "ce n'est pas diminuer la plénitude de la puissance apostolique: l'Océan même a ses bornes dans sa plénitude et s'il les outrepassait sans mesure aucune, sa plénitude serait un déluge, qui ravagerait tout l'univers ${ }^{61}$ '.

François de Laval aurait-il désavoué de tels propos et se serait-il élevé contre une telle conception de la dignité épiscopale? Il est permis d'en douter. Il avait, comme ses collègues de France, une très haute idée de son office de pasteur et de juge. Il était, comme eux, très dévoué au roi et aux intérêts de la couronne.

$57 \mathrm{P}$. Blet, Le clergé de France, II : 411.

58 Id., Les Assemblées du clergé, 553-554.

58 Id., Le clergé de France, II: 401.

60 Ibid.: 402.

61 P. Blet, Les Assemblées du clergé, 276. 
Et il était bon Français, tout aussi soucieux qu'ils l'étaient de voir maintenues et respectées les "libertés gallicanes".

Il reprochera amèrement, en 1674, à son ami Gazil d'avoir négligé "les privilèges de l'Eglise gallicane" dans le texte des bulles que ce dernier avait soumises à Rome en son nom ${ }^{62}$. La réponse de Gazil mérite d'être citée in extenso, car elle en dit long sur le "gallicanisme" des milieux que fréquentait François de Laval et sur la conception qu'on s'y faisait des "libertés gallicanes".

Nous ne disons pas que nous ayons des privilèges en notre Eglise gallicane, car le mot de privilège est odieux et il a été censuré dans une thèse de la Faculté par M. l'Avocat général et par d'autres. La raison est que si nous prétendons que ces droits sont des privilèges, il s'ensuit qu'en quelques cas les papes y pourraient déroger et que nous les tiendrions de la pure grâce du Saint-Siège. Au contraire nous estimons que ces libertés et franchises ne sont autre chose que le droit commun, dans l'usage duquel l'Eglise de France s'est maintenue contre les entreprises de la cour de Rome, qui avance toujours son autorité et la pousse par des progrès insensibles tant qu'elle peut. Or on n'a rien fait dans l'affaire de Québec qui empêche que l'Eglise canadienne se maintienne dans le droit commun de l'Eglise gallicane. [...]. L'Eglise de Québec ne sera pas plus sujette aux dispositions extraordinaires de la cour de Rome que toutes nos métropoles de France qui relèvent immédiatement de Rome, comme sont Lyon, Rouen, Bourges dont les appels vont à Rome et le Pape par les concordats nomme des juges in partibus. Voilà notre usage et ce sera celui du Canada ${ }^{63}$.

Les vues de Gazil n'ont rien d'un théoricien. Loin de là. Mais si l'on peut encore appeler "gallicanisme" ce sentiment qu'il exprime - et qui devait exister à des milliers d'exemplaires dans le royaume à l'époque - d'une tradition d'Eglise propre à la France et qu'il importe de sauvegarder et de maintenir pour le plus grand bien de la religion, on ne voit pas pourquoi on ne pourrait pas dire de François de Laval qu'il était "gallican", pourvu que l'on donne à ce terme non pas le sens "étroit" que lui imposera le XIXe siècle, mais le sens plus "diffus" - et plus conforme, croyons-nous, à la réalité du XVIIe siècle, - d'un attachement profond et presque instinctif à un certain concept et à une certaine expérience d'Eglise fondés sur le respect du

62 Altera Nova Positio: 341.

63 Ibid.: 341-342. 
passé et sur la volonté ferme de défendre ce passé contre les empiétements d'un pouvoir centralisateur ${ }^{64}$.

Certes, il existe au XVIIe siècle un certain nombre de prélats - un Maurice Le Tellier, un Gilbert de Choiseul, un Jacques Bossuet, pour ne nommer que les plus importants - qui sont, en ces matières, partisans, à divers degrés, des principes mis en avant par les théoriciens gallicans, mais il semble bien que, dans leur ensemble, les évêques de France soient d'un gallicanisme beaucoup plus "viscéral", beaucoup plus pratique, exprimant moins "une doctrine incontestable et unanimement reçue ${ }^{65}$ " qu'un sentiment instinctif de méfiance à l'endroit de tout ce qui paraîtrait menacer leurs propres pouvoirs et les traditions de leur Eglise. C'est à cet "ensemble" qu'appartient François de Laval.

\section{Le jansénisme}

L'emploi du mot jansénisme, nous l'avons signalé plus haut, date du milieu du XVIIle siècle. Mais, à l'époque, ce vocable désigne la seule "doctrine de Jansénius", c'est-à-dire le "système théologique de Jansénius, Evêque d'Ypres, sur la grâce et la prédestination " ${ }^{66}$ ". Dans l'Encyclopédie, un siècle plus tard, il prend le sens de "dispute sur la grâce, et sur les différents autres points de la doctrine chrétienne, à laquelle un ouvrage de Corneille Jansénius a donné lieu 67 ". La perpective est déjà plus large, mais elle est loin d'embrasser tout ce qu'il est convenu d'appeler aujourd'hui jansénisme. Ce mot a pris de fait, à partir du XIXe siècle, une telle extension qu'il est devenu presque impossible de lui donner "un contenu intellectuel précis ${ }^{68}$ ". C'est qu'

entre l'augustinisme rigide et archaïsant de Jansénius, la spiritualité pratique, bérullienne, plus encore qu'augustinienne de Saint-Cyran, les vues nuancées et imprégnées de thomisme que professent Arnaud et Nicole, mais que le P. Quesnel colore fortement de gallicanisme et de richérisme, et enfin l'obstination politique et partisane des appelants, il y a un écart considérable que seule dissimule la continuité historique ${ }^{69}$.

64 C'est le dénominateur commun que le $\mathrm{P}$. Congar assigne aux diverses formes de gallicanisme. Voir à ce sujet Y. Congar, "Gallicanisme", dans Catholicisme, IV : col. 1736.

65 P. Blet, Les Assemblées du clergé, 585.

66 Dictionnaire de Trévoux, III (Paris 1721) : col. 786.

67 Encyclopédie, VIII (Paris 1765) : 448.

$68 \mathrm{~L}$. Cognet, Le jansénisme (Paris 1961), 123.

69 Ibid. 
Louis Cognet, un des meilleurs spécialistes de la question, reconnaît que les seuls communs dénominateurs du mouvement sont, d'une part, "la conception (que les jansénistes avaient) d'un christianisme profondément exigeant, qui veut être vécu sans compromissions ni concessions" et, d'autre part, "une concience intense (qu'ils avaient tous en général) des droits de la personne, et surtout de la pensée personnelle, en face des absolutismes de l'autorité 70 ". Evidemment les condamnations pontificales aux XVIIe et XVIIIe siècles atteignent des doctrines précises, tirées d'auteurs et d'ouvrages connus, qu'on ne se fait d'ailleurs pas faute d'identifier, mais lorsque les contemporains s'en prennent au parti janséniste, ils ont en tête un certain nombre de stéréotypes qui ne sont pas nécessairement ceux contre lesquels s'élève le magistère.

Laissons de côté les savants traités de l'époque sur les problèmes de grâce et de liberté que seule une élite lisait et était à même de comprendre, et tenons-nous en plutôt à l'image que le public moyen pouvait se faire du jansénisme. Pour les uns, ceux généralement qui étaient du côté du pouvoir, les jansénistes avaient la réputation d'être des esprits rebelles et malintentionnés qu'il fallait à tout prix mettre à la raison ${ }^{71}$. Pour d'autres, ils étaient de subtils dialecticiens qui avaient, selon les uns, le mérite, selon les autres, le tort de dénoncer les transformations et les adaptations dont le message chrétien avait fait l'objet au cours des derniers siècles. Pour d'autres enfin, ils étaient tout simplement des hommes d'une grande sévérité et austérité de mœurs qui, pour cette raison, se posaient en adversaires déclarés des Jésuites qui avaient, selon eux, le tort d'engager l'Eglise sur la pente du laxisme. "Hommes de vertu et de piété éminentes et qui n'aiment pas les Jésuites", dira d'eux Agostino Favoriti, l'intime collaborateur d'Innocent XI ${ }^{72}$.

Certaines de ces définitions sont évidemment trop simplistes, voire trop peu "sérieuses ${ }^{73}$ ", pour que nous puissions les utiliser comme critères d'orthodoxie ou de non-orthodoxie à l'époque. Si nous nous en tenions à celle de Favoriti, par exemple, nous nous verrions dans l'obligation de conclure que Jean de Bernières et son disciple, François de Laval, n'étaient séparés des jansénistes que par l'amitié qu'ils vouaient aux Jésuites. Ce qui serait pour le moins paradoxal. Mais les définitions que nous venons

70 Ibid., 124.

${ }^{71}$ A. Adam, Du mysticisme à la révolte (Paris 1968), 230.

72 Cité par le même, 279.

73 Voir à ce propos le Dictionnaire de Trévoux, III : col. 786-787. 
d'évoquer, dans leur simplicité même, qui n'est pas sans trahir une certaine vérité, nous mettent sur la piste de préoccupations typiques du XVIIe siècle et qui peuvent permettre d'expliquer l'intelligence que l'on avait du jansénisme à l'époque et les attitudes qu'en conséquence on finissait par adopter à son endroit.

Pous nous en limiter aux milieux représentatifs de l'épiscopat et du clergé du XVIIe siècle, qui sont ceux dans lesquels évoluera François de Laval, signalons l'attitude d'un François Pallu, par exemple, confrère d'études et ami intime de l'évêque de Québec, dont la carrière, d'ailleurs, offre un parallèle frappant avec celle du prélat canadien.

Pallu, accusé vers 1680 d'être janséniste, se défend avec vigueur, assurant qu'il a "toujours affecté à Rome de paroistre fort éloigné, comme je le crois en effet, du jansénisme, et de toutes les personnes qui en sont soupçonnées ${ }^{74}$ ". Mais, quelque temps auparavant, dans une lettre aux directeurs du Séminaire des Missions Etrangères, il reconnaissait qu'il avait une morale très sévère et que ce qui l'empêchait d'être janséniste, c'était sa parfaite soumission aux décisions de Rome ${ }^{75}$.

L'aveu est significatif et il reflète assez bien la réaction de la plupart des prélats de l'époque face au jansénisme. Ce que l'on reproche au parti, ce n'est pas tant ses maximes - car on serait probablement d'accord avec un certain nombre d'entre elles -, mais c'est le fait qu'au nom de ces maximes les membres du parti soient prêts à mettre en péril tout l'ordre établi. Leur tort, leur principal tort, il est là. C'est toute une conception de la société qui est impliquée ici, conception que François Pallu et François de Laval partagent avec le haut-clergé de leur temps.

Pour la comprendre, il faut se reporter au concile de Trente et au célèbre débat sur la nature et l'extension des pouvoirs d'ordre et de juridiction dans l'Eglise. Deux concepts, à ce moment, s'affrontaient: l'un que l'on pourrait appeler hiérarchique et qui entendait donner ou redonner à l'Eglise une structure fondamentalement épiscopale; l'autre que l'on pourrait appeler missionnaire - élaborée surtout par Lainez, général des Jésuites - et qui visait à diminuer les pouvoirs épiscopaux et à majorer ceux du pape en vue de conférer à l'Eglise une plus grande flexibilité et une plus grande faculté d'adaptation dans un monde élargi et soumis de plus en plus au changement. Le concile

${ }^{74}$ Cité par L. Baudiment, François Pallu, principal fondateur de la Société des Missions Etrangères (1626-1684) (Paris 1934), 331, n. 1.

75 Ibid. 
refusa de trancher le débat, mais, de fait, grâce surtout à l'influence de Charles Borromée, champion et modèle de la réforme catholique en Europe, c'est le premier de ces concepts qui triompha, avec le résultat que les Eglises, en général, aux XVIIe et XVIIIe siècles, se tournèrent plutôt vers le passé que vers l'avenir, cherchant moins à s'adapter à des problèmes nouveaux qu'à maintenir ou à restaurer un ordre préétabli ${ }^{76}$. Cet ordre, c'était bien entendu, celui des sociétés européennes existantes, sociétés dont ces Eglises étaient partie prenante, au sein desquelles chacun de leurs membres avait sa place, son rôle, sa fonction, où les hiérarchies aussi bien civiles qu'ecclésiastiques étaient considérées comme autant de piliers d'un ordre immuable et, pour ainsi dire, sacré.

Pour le roi, pour l'épiscopat gallican, toute atteinte à cette structure est perçue comme une menace pour la société ellemême. D'où la rigueur avec laquelle on traite le parti janséniste dans la deuxième moitié du XVIIe siècle. Fait paradoxal, évêques et jansénistes étaient fondamentalement d'accord sur le concept d'Eglise illustré par Charles Borromée - Saint-Cyran et ses disciples, nous l'avons vu, s'étaient même fait sur ce point les alliés de la hiérarchie contre les prétentions des réguliers -, mais à partir du moment où le parti janséniste refusait de se soumettre aux autorités établies, les évêques n'avaient pas d'autre choix que de se tourner contre lui.

François de Laval, tout comme son collègue Pallu, partage entièrement cette vision de l'Eglise et de la société. Sa façon de traiter les Récollets de Nouvelle-France au cours des années 1670-1685 le montre bien ${ }^{77}$. N'eût été la grande amitié qui l'unissait aux Jésuites, qui nous dit qu'il n'aurait pas eu, un jour ou l'autre, avec ces derniers, de semblables conflits de juridiction? L'exemple de François Pallu en Indochine est là pour nous rappeler, s'il en était besoin, que les relations, à l'époque, étaient loin d'être faciles entre ces évêques missionnaires, tirés du clergé séculier, et leurs collaborateurs jésuites ${ }^{78}$.

Le sentiment de méfiance d'une bonne partie de l'épiscopat gallican à l'endroit du parti janséniste dans la deuxième moitié du XVIIe siècle semble donc provoqué beaucoup plus par les "désobéissances" du parti que par les idéaux dont il se réclame.

${ }^{76} \mathrm{~J}$. Bossy, Postface à H. O. Evennett, The Spirit of the CounterReformation (South Bend-Londres 1968), 135 et suiv.

77 Altera Nova Positio: 251 et suiv.

$78 \mathrm{H}$. Chappoulie, Aux origines d'une Eglise. Rome et les missions d'Indochine au XVIIe siècle, II (Paris 1948) : 41 et suiv. 
Souriau, qui s'est beaucoup intéressé au milieu de l'Ermitage de Caen, ne croit pas que l'évêque de Québec, hostile peut-être au jansénisme, "ait été l'adversaire des jansénistes eux-mêmes", car, nous dit-il, "il (songea) pendant quelque temps à céder son abbaye d'Estrée à une communauté de Bernardines et, pour cette affaire, il (fut) longtemps en correspondance avec l'abbesse de Port-Royal-des-Champs ${ }^{79}$ ". Prélat d'une rare abnégation et reconnu pour son rigorisme, il est probable que François de Laval avait quelque sympathie pour l'idéal de vie proposé par les jansénistes, mais il est vraisemblable de croire, en même temps, qu'il n'avait que peu d'intérêt et ressentait probablement quelque répugnance pour leurs spéculations théologiques, partageant en cela l'avis de saint François de Sales et de bon nombre de dévots en France à l'époque "qu'il valait mieux s'attacher à faire un bon usage de la grâce que d'en disputer ${ }^{80}$ ".

\section{L'ultramontanisme}

Si l'on peut parler d'un certain "gallicanisme" de François de Laval, s'il est difficile d'admettre qu'il ait été systématiquement antijanséniste, peut-on encore accepter l'affirmation de ses biographes selon laquelle il aurait été un ultramontain ?

Avant que de répondre à cette question, il importerait de savoir ce que ces mêmes biographes entendent par ultramontanisme. Ce vocable, nous l'avons vu plus haut, est une création du XIXe siècle. Il a, chez Littré, le sens très étroit de "doctrine de l'infaillibilité du pape ${ }^{81}$ ". De fait, c'est le sens que très souvent on donne au mot ultramontanisme à l'époque de Vatican I ${ }^{82}$. Roger Aubert, plus complet et plus nuancé, met sous ce même terme tout un corps de doctrine concernant, d'une part, les droits et privilèges spéciaux du pape, notamment sa primauté de juridiction sur l'ensemble de l'Eglise et son infaillibilité personnelle; d'autre part, les droits de l'Eglise par rapport à l'Etat, c'est-àdire, en gros, l'indépendance totale de l'Eglise par rapport à ce dernier en matières spirituelles et la dépendance au moins indirecte de celui-ci par rapport à l'Eglise dans certaines questions dites mixtes ${ }^{83}$.

79 M. Souriau, Le mysticisme en Normandie au XVIIe siècle, 368.

$80 \mathrm{~L}$. Cognet, Le jansénisme, 18.

81 Dictionnaire de Littré (Paris 1883), IV, 2: col. 2387.

82 P. Pecchiai, "Ultramontanismo", dans Enciclopedia Cattolica, XII (Cité du Vatican 1954) : col. 724.

83 R. Aubert, Vatican I (Paris 1964), 29. 
Le XVIIe siècle employait le mot ultramontain, soit comme adjectif, soit comme substantif, pour désigner l'attitude de ceux qui se proposaient "d'exalter sans cesse la grandeur du SaintSiège" et qui suivaient, en ces matières, les opinions des "canonistes italiens ${ }^{84 "}$. De fait, le terme sera si souvent employé par les jansénistes et régalistes au XVIIe siècle, par les joséphistes et fébronianistes, au siècle suivant, pour épingler leurs adversaires, qu'il finira par n'avoir plus que le sens, assez vague, de personne favorable au Saint-Siège ${ }^{85}$.

Les biographes de François de Laval estiment avoir de bonnes raisons de croire que celui-ci était ultramontain, c'est-àdire d'un grand attachement au pape et d'un grand respect pour les droits et prérogatives du Saint-Siège.

Ils insistent beaucoup sur le fait que leur héros entretenait une correspondance suivie avec la cour de Rome. Cela est vrai pour la période du vicariat apostolique, car la Nouvelle-France, en tant que territoire de mission, dépendait directement du Saint-Siège; ce ne l'est plus à partir de 1674, puisque "devenue [...] Eglise de plein droit", Québec communique "désormais avec Rome par les canaux ordinaires", ce qui signifie que la plupart des affaires sont "définitivement résolues en France et que celles qui vont à Rome ne passent plus obligatoirement par la Propagande", mais sont "attribuées aux diverses Congrégations selon les compétences particulières de celles-ci ${ }^{86}$ ". Ajoutons qu'il y a, à la même époque, nombre d'évêques français qui entretiennent avec Rome des rapports très étroits sans pour autant se considérer ou être considérés comme ultramontains ${ }^{87}$. Bien au contraire.

Toujours à l'appui de la même thèse, on a cherché à exploiter le fait que l'évêque de Québec sollicitait régulièrement de Rome certaines facultés ou, si l'on préfère, certains pouvoirs de juridiction spéciaux. Ces facultés, dites "missionnaires", étaient des pouvoirs que concédait la Congrégation de la Propagande à l'avantage de ceux qui étaient appelés à travailler auprès des infidèles, des hérétiques et des schismatiques ${ }^{88}$. Or Bernard Jacquelines, qui s'est beaucoup intéressé à cet aspect du travail de la

84 A. G. Martimort, Le gallicanisme de Bossuet, 15.

85 P. Pecchiai, loc. cit.

${ }^{56} \mathrm{~L}$. Campeau, "Les initiatives de la S. Congrégation en faveur de la Nouvelle-France", dans Sacrae Congregationis de Propaganda Fide Memoria Rerum, 1622-1972, I/2, 1622-1700 (Rome-Fribourg-Vienne 1972) , 793.

$87 \mathrm{P}$. Blet, Le clergé de France, II : 400-401.

88 L. Campeau, art. cit., 790-791. 
Propagande, a découvert qu'en France, à la même époque, de bons évêques gallicans, par dizaines, ne se faisaient aucun scrupule de demander à Rome les dites facultés ${ }^{89}$. Faudrait-il en faire, pour autant, eux aussi, des ultramontains ?

Les biographes de François de Laval insistent également sur le fait que l'évêque de Québec est responsable de l'introduction de la liturgie romaine en Nouvelle-France. Encore ici, rien de tellement original, puisque c'était l'usage suivi dans la grande majorité des diocèses français à l'époque. Ce n'est que vers la fin du XVIIe siècle, et au cours du XVIIIe, que les liturgies néo-gallicanes commenceront à faire leur apparition, puis s'imposeront progressivement un peu partout en France ${ }^{90}$.

Enfin, Auguste Gosselin, se fondant sur la correspondance de Mgr de Laval, ne craint pas d'affirmer que son héros était animé d'extraordinaires "sentiments de respect, d'affection et de dévouement ${ }^{91}$ " envers la cour de Rome. Et il ajoute: "A cette époque où les évêques de France étaient presque tous imbus d'idées plus ou moins gallicanes, on est dans l'admiration en voyant les sentiments de dévouement au Saint-Siège et de respect pour le Souverain Pontife qui respirent dans toutes ses lettres ${ }^{92}$." Fort bien, mais ces sentiments de respect et de déférence, on les retrouve chez la plupart des prélats français de l'époque, voire chez les plus gallicans d'entre eux, comme permet de le constater la lecture des ouvrages de Pierre Blet sur les Assemblées du clergé au XVIIe siècle ${ }^{93}$. François de Laval ultramontain ? Mais, alors, il faudrait admettre que tous ces évêques "imbus d'idées plus ou moins gallicanes", dont nous parle Gosselin, l'étaient, eux aussi. Cela aurait-il encore un sens ?

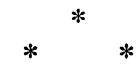

Il n'y a qu'une façon de surmonter le dilemme, c'est d'admettre que François de Laval, comme la plupart des prélats de son temps, échappe aux catégories commodes dans lesquelles les historiens du XIXe siècle ont cherché à l'enfermer. Ces catégo-

89 B. Jacquelines, "Missions en France", dans Sacrae Congregationis ..., $\mathrm{I} / 2,119-120$.

$90 \mathrm{~J}$. Gaillard, "Liturgies néo-gallicanes", dans Catholicisme, IV: col. 1728-1731.

91 A. Gosselin, Vie de Mgr de Laval, II : 283.

92 Altera Nova Positio: 900.

93 Nous renvoyons tout simplement aux deux ouvrages en question utilisés abondamment tout au long de cette étude. 
ries, nous l'avons vu, sont trop étrangères à la réalité vivante et mouvante du XVIIe siècle, trop liées aux intérêts et aux préoccupations de ceux qui les ont élaborées au XIXe, trop aériennes, pour que nous puissions les appliquer sans discernement à l'infinie variété des idées et des sentiments qui existaient, en ces domaines, dans la France du XVIIe siècle.

En un sens, on pourrait peut-être dire, pour corriger l'image un peu simpliste qu'ont tracée de lui ses biographes, que François de Laval était moins antijanséniste, moins ultramontain et plus gallican qu'on ne l'a prétendu. Mais ce serait encore là tomber dans le piège que, consciemment ou non, ils nous ont tendu. Le plus vrai ne serait-il pas encore de reconnaître que François de Laval échappe en grande partie à ces catégories; qu'à l'instar de beaucoup de prélats français de son époque qui, on l'oublie très souvent, n'étaient ni théologiens de métier ni juristes de profession, bien plus, qui craignaient les subtilités des premiers et les intransigeances des autres; qu'à leur exemple, il n'aspirait qu'à une chose: exercer en toute liberté, dans les limites de son diocèse, sous la double protection du pape et du roi, également respectés, ses fonctions de pasteur et de juge, conformément aux traditions de l'Eglise gallicane et dans le respect des normes et de l'idéal tridentins. Tout le reste est supposition. 